\title{
Cardiometabolic Phenotype and Arterial Stiffness in HIV-Positive Black African Patients
}

\author{
Daniel Lemogoum ${ }^{*}$, William Ngatchou1, Philippe Van De Borne1, Valérie Ndobo², \\ Marc Leeman', Euloge Yagnigni' ${ }^{2}$, Emiline Tiogou ${ }^{2}$, Elisabeth Nga $^{2}$, Charles Kouanfack ${ }^{2}$, \\ Luc Van Bortel ${ }^{3}$, Jean-Paul Degaute ${ }^{1}$, Michel P. Hermans ${ }^{4}$ \\ ${ }^{1}$ Hypertension Clinic, Erasme University Hospital, Brussels, Belgium \\ ${ }^{2}$ Department of Cardiology, Central Hospital, Yaounde, Cameroon \\ ${ }^{3}$ Heymans Institute of Pharmacology, Ghent University, Ghent, Belgium \\ ${ }^{4}$ Department of Endocrinology and Nutrition, Cliniques Universitaires St-Luc, Brussels, Belgium \\ Email: "dlems2002@yahoo.fr
}

Received 28 January 2014; revised 21 March 2014; accepted 31 March 2014

Copyright (C) 2014 by authors and Scientific Research Publishing Inc.

This work is licensed under the Creative Commons Attribution International License (CC BY).

http://creativecommons.org/licenses/by/4.0/

(c) (i) Open Access

\section{Abstract}

Objective: To test the hypothesis that Human Immunodeficiency Virus (HIV) infection in Black patients is associated with increased cardiometabolic risk factors that may increase aortic stiffness assessed by pulse wave velocity (PWV). Methods: We matched 96 Cameroonian Controls to 238 (Un) treated HIV-positive patients [HIV] for age. In each participant, aortic PWV (ComplioR), blood pressures (BP), lipid profile and fasting blood glucose (FPG) were measured. Results: Waist circumference was lower in HIV than in Controls (both $p<0.001$ ). The prevalences of impaired FPG

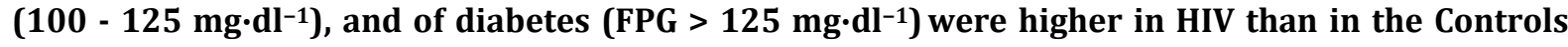
(50\% vs $27 \%$, and $23 \%$ vs $1 \%$, respectively; both $p<0.0001$ ). HDL-C was lower in HIV as compared to the Control $(p=0.02)$. Fasting triglycerides (TG) and the atherogenic dyslipidemia ratio $[\log (\mathrm{TG}) / \mathrm{HDL}-\mathrm{C}]$ were higher in HIV than in the Controls (both $p<0.05)$. Hypertension prevalence was high but comparable in the two groups $(48 \%$ vs $44 \%$ respectively, $p>0.05)$. HIV patients exhibited a twice-higher prevalence of MetS (AHA/NHLBI score $\geq 3 / 5$ ) than that of the Controls $(41 \%$ vs $21 \% ; p<0.01$ ). Similarly, severity of MetS phenotype was higher in HIV as compared to the Controls $(2.14 \%$ vs $1.59 \% ; p<0.0001)$. PWV adjusted for age, mean BP and gender was faster in $\mathrm{HIV}$ in comparison to the controls $(7.33 \mathrm{~m} / \mathrm{s}$ vs $6.86 \mathrm{~m} / \mathrm{s}$, respectively; $p=0.036)$. Conclusions: HIV infection is associated with higher prevalence of MetS and its phenotype in Black African patients that may induce increased aortic stiffness.

"Corresponding author. 


\section{Keywords}

\section{Metabolic Syndrome; Pulse Wave Velocity; Arterial Stiffness; Human Immunodeficiency Virus Infection; Black Cameroonian Patients}

\section{Introduction}

Human immunodeficiency virus (HIV) infection and its therapy are associated with increased risk of cardiovascular diseases (CVD), such as myocardial infarction [1] and stroke [2]. The underlying mechanisms remain insufficiently understood. Early-onset atherosclerosis in the pathophysiology of HIV-related CVD has been linked to systemic low-grade inflammatory state triggered by both HIV and opportunistic HIV-related infections [3][7], and to adverse cardiometabolic effects of current antiretroviral therapy (ART) [5]-[8]. Chronic infection in HIV-individuals promotes chronic arterial inflammation, which in turn, induces endothelium dysfunction and atherosclerosis [6] [7]. Endothelial dysfunction was proposed as a plausible link between HIV infection and atherosclerosis [4] [5] [7]-[9].

Over the last two decades, availability of ART, particularly protease inhibitors (PIs), changed the course of HIV infection, leading to marked reduction in morbidity and mortality [10] and increased patient's life expectancy. However, prolonged exposure to HIV and ART led to new clinical and public health challenges, such as increased cardiometabolic abnormalities including visceral fat accumulation, diabetes, dyslipidemia, insulin resistance (IR), and hypertension [8] [11] [12]. All these abnormalities are either individual components of the metabolic syndrome (MetS) phenotype or of its underlying pathophysiology, and may predispose to the development of atherosclerosis, whereas westernized, unhealthy lifestyles and obesity are causally related to acquisition of a MetS phenotype.

Increased arterial stiffness assessed by aortic pulse wave velocity (PWV) is a marker of atherosclerosis and an independent predictor of cardiovascular (CV) outcomes [13]. PWV was reported to be accelerated in Caucasians HIV-infected individuals in comparison to healthy controls [9] [14]. Whether similar changes occur in Black African patients born and living in sub-Saharan Africa (SSA) is unclear. Despite the prevalence of HIV infection being highest in SSA [15], the related cardiometabolic disorders and atherosclerosis risk has been poorly explored in native populations with HIV as yet little exposed to westernized lifestyles. We therefore hypothesized that HIV infection in Black African patients is associated with increased burden of cardiometabolic risk factors that may accelerate arterial structural damage which translates into early-onset increased arterial stiffness.

\section{Methods}

The study design was cross-sectional including 334 volunteer subjects aged $>18$ years recruited from the HIV clinic of Yaoundé Central Hospital (Cameroon) between September 2009 and May 2010. Two groups were analyzed in parallel: a group of 96 apparently healthy control subjects [Controls] was compared to a group of 238 HIV-positive patients, both treated $(n=130)$ or untreated $(n=108)$ with antiretroviral drugs [HIV], with comparable mean (1SD) age (41 \pm 12 [Controls] vs $40 \pm 11$ [HIV]). Inclusion criteria for patients were serologically-documented HIV infection; and for Controls, a recently-documented (within past year) seronegative status for HIV. Exclusion criteria for Controls and HIV patients included: documented CVD and/or conventional CV risk factors like hypertension, dyslipidemia, diabetes, smoking, and/or current use of anti-diabetics, antihypertensive or any others CV drugs. Each volunteer signed an informed consent, and the study was approved by the Institutional Review Board of the Yaoundé Central Hospital and Cameroonian Ministry of Public Health.

For each study participant socio-demographic and clinical variables were recorded including age, sex, duration since HIV diagnosis (as surrogate for HIV infection duration), ART history and duration, lifestyle habits (cigarette smoking, alcohol consumption), history of hypertension, diabetes, dyslipidemia, use of anti-diabetic, antihypertensive or lipid-lowering drugs. All participants underwent physical examination by a single operator (DL) who measured the following parameters: weight, height, body mass index (BMI), waist circumference (as proxi for visceral fat), hip circumference, waist-to-height ratio and conicity index [waist circumference (m)/ $0.109 \sqrt{[}$ weight $(\mathrm{kg}) /$ height $(\mathrm{m})]$, the latter two being validated surrogates for central adiposity distribution (reference values for conicity index, from lean controls $(n=79$ ) with normal glucose tolerance (NGT): $1.22 \pm 0.09$ 
$\mathrm{m}^{2} / \mathrm{kg}$ (mean $\pm 1 \mathrm{SD}$ ); $1.16-1.26 \mathrm{~m}^{2} / \mathrm{kg}$ [interquartile range] and $1.03-1.43 \mathrm{~m}^{2} / \mathrm{kg}$ [range]).

Hypertension was defined as systolic BP $\geq 140 \mathrm{mmHg}$ and/or diastolic BP $\geq 90 \mathrm{mmHg}$, and/or current treatment with BP-lowering medication(s) [16]. The presence of MetS was defined according to AHA/NHLBI criteria (score $\geq 3 / 5$ ), with mean MetS score used as surrogate for whole-body insulin resistance [17].

\subsection{Biochemical Parameters}

In all participants, a venous blood sample was collected after an overnight fast to measure the concentration of the following biochemical variables: fasting plasma glucose (FPG), lipids (total cholesterol (C), HDL-C, triglycerides (TG); LDL-C (computed from Friedewald's formula), and non-HDL-C (by subtracting HDL-C from total C). NGT, impaired fasting glucose (IFG) and diabetes were defined as FPG $<100 \mathrm{mg} \cdot \mathrm{dL}^{-1}, 100-125$ $\mathrm{mg} \cdot \mathrm{dL}^{-1}$, and $>125 \mathrm{mg} \cdot \mathrm{dL}^{-1}$, self-reported diagnosis or use of antidiabetic medications, respectively. The prevalence of atherogenic dyslipidemia was defined as the combination of low HDL-C ( $<40$ [males] and $<50 \mathrm{mg} \cdot \mathrm{dL}^{-1}$ [females]) plus high fasting TG $\left(\geq 150 \mathrm{mg} \cdot \mathrm{dL}^{-1}\right)$, whereas atherogenic dyslipidemia severity, as continuous variable, was determined by the $\log (\mathrm{TG}) / \mathrm{HDL}-\mathrm{C}$ ratio [normal values for the latter, obtained in 79 lean healthy subjects without familial histories for diabetes or early-onset CVD: 0.036 (mean); 0.012 (SD); 0.014 (minimum); 0.067 (maximum)] [18]. Urinary $\mathrm{Na}^{+}$and urinary $\mathrm{K}^{+}$were obtained from urine spots. All samples were analyzed at the biochemical laboratory of ULB-Erasme Hospital, Brussels, Belgium.

\subsection{HIV History}

Plasma CD4+ cells counts (most recent value and nadir whenever possible), and cumulative exposure to nonnucleoside reverse-transcriptase inhibitors (NNRTI), nucleoside reverse-transcriptase inhibitors (NRTIs), and PIs were recorded.

\subsection{Hemodynamic Measurements}

To ensure steady state, all hemodynamic measurements were performed in the morning after 15 minutes of supine rest in a quiet room. All participants were instructed to abstain from strong physical efforts, and drinking caffeine, tea, or any alcoholic beverage for at least 2 hours before hemodynamic measurements. All measurements were performed by the same investigator in triplicate, and averaged for analysis.

\subsection{Brachial Blood Pressures and Heart Rate}

Brachial systolic and diastolic blood pressure (BP) and heart rate (HR) were recorded at the right arm with appropriate cuff size using automated sphygmomanometer (HEM-705 CP, Omron Corporation, Tokyo, Japan). Pulse pressure (PP) was calculated as systolic minus diastolic BP, and mean arterial pressure (MAP) as diastolic BP plus one-third of PP.

\subsection{Aortic Pulse Wave Velocity}

Aortic pulse wave velocity (PWV) was measured by the same investigator (DL) using sequential recordings of the arterial pressure waveform at the carotid and femoral arteries using a validated [19] noninvasive device (ComplioR, Artech Medical, France), which performs pressure wave recordings and automatically calculates PWV. The distance was defined as: distance from the supra-sternal notch to femoral artery minus distance from carotid artery to supra-sternal notch. Pulse transit time was averaged over 10 consecutive beats. PWV corresponds to the ratio of the distance (meters) to the transit time (seconds). Intra-observer coefficient of variation for aortic PWV for the same investigator (DL) is $3.8 \% \pm 1.3 \%$ [20].

\section{Statistical Methods}

Data are presented as means ( \pm 1 standard deviation (SD)) or as proportions (\%). The significance of differences between means was assessed by Student's $t$ test or by alternate Welch's test for data sets with significant differences in SDs, and by Fisher's Exact test for differences in proportions, with TG values log-transformed prior to statistical analysis. A multiple regression analysis was performed to assess the independent determinant of the PWV. All relevant variables that correlated with PWV were included in that model. Results were considered 
significant for a $p<0.05$ and, or non-significant (NS) for $p \geq 0.05$, respectively.

\section{Results}

\subsection{Controls and Patients' Characteristics}

Gender distribution was not statistically different between Controls and HIV patients (male-to-female ratio 28:72 [Controls] and 24:76 [HIV]). Body height was also comparable between groups, whereas body weight was $10.0 \mathrm{~kg}$ lower in HIV ( $p<0.0001)$ as compared to Controls. As consequence, body mass index was markedly lower in HIV than in Controls $(p=0.0002)$. Waist circumference was lesser, by an average $7 \mathrm{~cm}$ in HIV compared to Controls $(p<0.0001)$. Conicity index and waist-to-hip ratio were comparable in the two groups, whereas waist-to-height ratio, another surrogate for central adiposity was lower in HIV than in Controls ( $p=$ 0.0001; Table 1).

Table 2 describes the cardiometabolic phenotype of the two study groups.

Based on FPG, the proportion of participants with normal glucose homeostasis (NGT) was higher in Controls than in HIV, while the prevalences of IFG and of diabetes were markedly increased in HIV (both $p<0.0001$ ). Atherogenic dyslipidemia prevalence was higher in HIV than in Controls, although this difference did not reach statistical significance. HIV patients exhibited a higher prevalence of MetS than Controls $(p<0.01)$. In addition, as shown on Figure 1, the severity of the MetS, i.e. the score of its discrete phenotype's components based on AHA/NHLBI, was more pronounced in HIV than in Controls, indicative of decreased whole-body insulin sensitivity $(p=0.001)$.

\subsection{Laboratory Values}

Mean FPG was markedly higher in HIV than in Controls ( $p<0.0001)$. Total cholesterol (C), LDL-C and nonHDL-C were comparable between groups. HDL-C was $5 \mathrm{mg} \cdot \mathrm{dL}^{-1}$ lower in HIV than in Controls $(p=0.02)$. The atherogenic ratios [total C/HDL-C] and [non-HDL-C/HDL-C], the atherogenic dyslipidemia ratio [log(TG)/ HDL-C], and fasting TG were significantly higher in HIV (all $p<0.05$ ). Urinary $\mathrm{Na}^{+}$concentration was lower

\begin{tabular}{|c|c|c|c|c|}
\hline & & controls & HIV & $p$ \\
\hline$n$ & & 96 & 238 & $\sim$ \\
\hline sex ratio (M:F) & $\%$ & $28: 72$ & $24: 76$ & $N S$ \\
\hline age & years & $41(12)$ & $40(11)$ & NS \\
\hline duration since HIV diagnosis & months & $\sim$ & $34(32)$ & $\sim$ \\
\hline HAART duration & months & $\sim$ & $33(27)$ & $\sim$ \\
\hline CD4+ T lymphocytes & $\mu \mathrm{L}^{-1}$ & $\sim$ & 343 (201) & $\sim$ \\
\hline weight & $\mathrm{kg}$ & $75.3(15.4)$ & $65.3(12.3)$ & $<0.0001$ \\
\hline height & $\mathrm{m}$ & $1.64(0.09)$ & $1.63(0.09)$ & NS \\
\hline BMI & $\mathrm{kg} \cdot \mathrm{m}^{-2}$ & $28.1(5.9)$ & $25.0(8.7)$ & 0.0002 \\
\hline $\mathrm{BMI}<27.5 \mathrm{~kg} \cdot \mathrm{m}^{-2}$ & $\%$ & 51 & 75 & $<0.0001$ \\
\hline waist circumference & $\mathrm{cm}$ & $90(13)$ & $83(10)$ & $<0.0001$ \\
\hline hip circumference & $\mathrm{cm}$ & $112(26)$ & $108(24)$ & NS \\
\hline waist $\cdot h i p^{-1}$ & $\sim$ & $0.84(0.22)$ & $0.80(0.18)$ & NS \\
\hline conicity index & $\mathrm{m}^{2} \cdot \mathrm{kg}^{-1}$ & $1.24(0.24)$ & $1.22(0.18)$ & NS \\
\hline waist height ${ }^{-1}$ & $\sim$ & $0.55(0.09)$ & $0.51(0.07)$ & 0.0001 \\
\hline
\end{tabular}

Results expressed as means (1SD) or proportions (\%). BMI: body mass (Quetelet's) index; F: female; HAART: highly-active antiretroviral therapy; HIV: human immunodeficiency virus; M: male. NS: non-significant. 
Table 2. Cardiometabolic phenotype.

\begin{tabular}{|c|c|c|c|c|}
\hline & & controls & HIV & $p$ \\
\hline$n$ & & 96 & 238 & $\sim$ \\
\hline \multicolumn{5}{|l|}{ glucose homeostasis } \\
\hline normal & $\%$ & 72 & 28 & $<0.0001$ \\
\hline impaired fasting glucose & $\%$ & 27 & 50 & $<0.0001$ \\
\hline diabetes & $\%$ & 1 & 22 & $<0.0001$ \\
\hline atherogenic dyslipidemia & $\%$ & 3 & 9 & NS \\
\hline metabolic syndrome & $\%$ & 21 & 41 & 0.0010 \\
\hline metabolic syndrome score & $0 / 5$ to $5 / 5$ & $1.59(1.02)$ & $2.14(1.05)$ & $<0.0001$ \\
\hline $0 / 5$ score & $\%$ & 14 & 5 & 0.0128 \\
\hline $1 / 5$ score & $\%$ & 35 & 24 & 0.0298 \\
\hline $2 / 5$ score & $\%$ & 29 & 30 & NS \\
\hline $3 / 5$ score & $\%$ & 20 & 33 & 0.0232 \\
\hline 4/5 score & $\%$ & 2 & 8 & NS \\
\hline $5 / 5$ score & $\%$ & 0 & 0 & NS \\
\hline
\end{tabular}

Results expressed as means (1SD) or proportions (\%). HIV: human immunodeficiency virus. NS: non-significant.

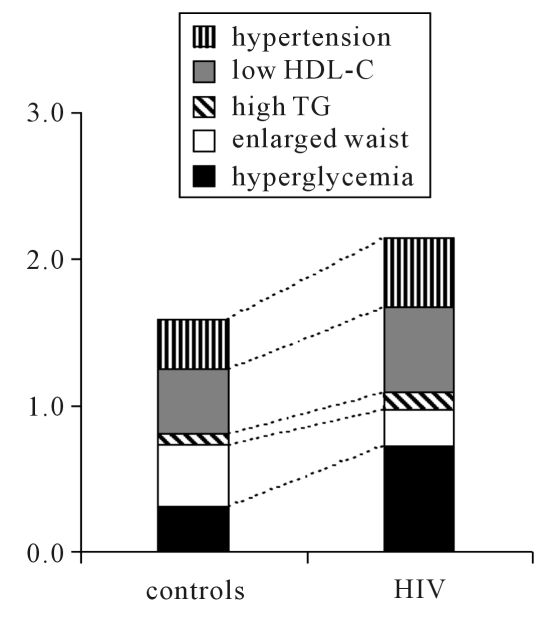

Figure 1. Metabolic syndrome score (0/5 to 5/5; columns' height) and cohort-adjusted distribution of the five individual components of the metabolic syndrome phenotype to the score in Controls $(n=96$; left bars) compared to those of (un)treated HIV patients ( $n=238$; right bars). HDL-C: high-density lipoprotein cholesterol; TG: triglycerides. For differences between groups: $p<0.0001$ (metabolic syndrome score); $p<0.001$ (hyperglycaemia); $p=0.0015$ (enlarged waist); $p$ NS (high triglycerides); $p=0.0298$ (low HDL-C) and $p=$ 0.0380 (high blood pressure).

in HIV than in Controls ( $p=0.002$ ), whereas urinary $\mathrm{K}^{+}$did not differ between groups (Table 3 ).

\subsection{Hemodynamic Parameters}

The prevalence of hypertension was high and comparable in the two groups, despite exclusion of participants with a known history of (un)treated hypertension. MAP was significantly lower in HIV than in Controls ( $p<$ 0.039). Heart rate was 8 b.p.m higher in HIV than in Controls $(p<0.0001)$. Age, mean BP and gender-adjusted 
Table 3. Laboratory values.

\begin{tabular}{|c|c|c|c|c|}
\hline & & controls & HIV & $p$ \\
\hline$n$ & & 96 & 238 & $\sim$ \\
\hline fasting glycaemia & $\mathrm{mmol} \cdot \mathrm{l}^{-1}$ & $5.2(0.9)$ & $6.5(2.4)$ & $<0.0001$ \\
\hline total cholesterol & $\mathrm{mg} \cdot \mathrm{dl}^{-1}$ & $167(43)$ & 164 (49) & NS \\
\hline LDL-C & $\mathrm{mg} \cdot \mathrm{dl}^{-1}$ & $103(34)$ & 103 (38) & $N S$ \\
\hline non-HDL-C & $\mathrm{mg} \cdot \mathrm{dl}^{-1}$ & 118 (39) & 122 (39) & $N S$ \\
\hline HDL-C & $\mathrm{mg} \cdot \mathrm{dl}^{-1}$ & $49(17)$ & $44(21)$ & 0.0244 \\
\hline total-C.HDL-C ${ }^{-1}$ & $\sim$ & $4.11(2.95)$ & $5.19(5.18)$ & 0.0173 \\
\hline non-HDL-C.HDL-C ${ }^{-1}$ & $\sim$ & $3.06(2.96)$ & 4.19 (5.18) & 0.0129 \\
\hline TG & $\mathrm{mg} \cdot \mathrm{dl}^{-1}$ & $67(35)$ & 118 (293) & $<0.0001^{\S}$ \\
\hline $\mathrm{TG}<150 \mathrm{mg} \cdot \mathrm{dl}^{-1}$ & $\%$ & 94 & 89 & NS \\
\hline $\log (\mathrm{TG}) \cdot \mathrm{HDL}^{-\mathrm{C}^{-1}}$ & $\sim$ & $0.048(0.044)$ & $0.077(0.121)$ & 0.0015 \\
\hline urinary $\mathrm{Na}^{+}$ & $\mathrm{mmol} \cdot \mathrm{l}^{-1}$ & 116 (59) & $96(52)$ & 0.0024 \\
\hline urinary $\mathrm{K}^{+}$ & $\mathrm{mmol} \cdot \mathrm{l}^{-1}$ & 74 (32) & $71(34)$ & NS \\
\hline
\end{tabular}

Results expressed as means (1SD) or proportions (\%). ${ }^{\S}$ From $\log (\mathrm{TG}): 1.78(0.19)$ [controls] and $1.92(0.26)$ [HIV]; HDL: high-density lipoprotein; HIV: human immunodeficiency virus; LDL: low-density lipoprotein; TG: triglycerides. NS: non-significant.

PWV was faster in HIV than in controls $(p=0.036$ ), indicating increased arterial stiffness, and predictive of higher atherosclerosis risk in HIV (Table 4). Linear regression analysis showed significant correlations between aortic PWV and weight (slope $-0.90 ; R^{2}: 0.0136 ; p=0.040$ ); LDL-C (slope 3.26; $R^{2}: 0.0276 ; p=0.008$ ); HDL-C (slope 1.44; $R^{2}: 0.0185 ; p=0.030$ ); and non-HDL-C (slope 3.06; $R^{2}: 0.0222 ; p=0.018$ ). There were no significant correlations between PWV and other relevant variables.

\subsection{Characteristics of Treated vs Untreated HIV Patients}

Age, gender distribution, FPG, prevalence of abnormal glucose homeostasis and of MetS, MetS score, heart rate and $\mathrm{CD}_{4}^{+}$lymphocytes count were not statistically different between treated and untreated HIV patients (not shown). The prevalence of hypertension, systolic BP and MAP were higher in treated HIV patients (all $p<0.05$ vs untreated patients). In HIV-treated patients, waist circumference was significantly larger $(p=0.002)$, and the waist height ${ }^{-1}$ ratio also greater $(p=0.02)$ than in untreated HIV patients. In HIV-treated patients, ART duration, averaging 33 (27) months, was not associated with arterial stiffness ( $P=N S$ ). Mean total cholesterol and LDL-C levels were higher in treated HIV patients (both $p<0.01$ ), who also exhibited higher HDL-C, lower atherogenic ratios (total C.HDL- $\mathrm{C}^{-1}$ and non-HDL-C.HDL- $\mathrm{C}^{-1}$ ), as well as lower atherogenic dyslipidemia ratio than untreated HIV patients (all $p<0.001$ ) (Table 5).

\section{Discussion}

The key finding of this study is that native Black African HIV-positive patients living in sub-Saharan Africa are at increased risk of CVD. Thus, abnormal aortic compliance translating into increased aortic stiffness was observed, alongside a high prevalence and burden of MetS and its individual components, such as hypertension, diabetes, impaired fasting glucose, low HDL-C and high TG, many of which represent established or emerging, lifestyle-modifiable CV risk factors. In spite of sub-Saharan Africa being most severely affected by HIV infection, the authors are not aware of any previous report on the cardiometabolic phenotype and aortic distensibility of Black African HIV-positive patients.

With respect to hypertension among HIV-positive individuals, little is known about its epidemiology in sub-Saharan Africa. The present study observes a higher prevalence of elevated blood pressure among the whole 
Table 4. Blood pressure and arterial function.

\begin{tabular}{lcccc}
\hline \multicolumn{1}{l}{} & & controls & HIV & $p$ \\
\hline$n$ & & 96 & 238 & $\sim$ \\
hypertension & $\%$ & 44 & 48 & NS \\
\multicolumn{1}{c}{ systolic } & $\%$ & 2 & 7 & $N S$ \\
$\quad$ diastolic & $\%$ & 15 & 17 & $N S$ \\
$\quad$ systolo-diastolic & $\%$ & 27 & 25 & NS \\
SBP & $\mathrm{mm} \mathrm{Hg}$ & $128(24)$ & $124(27)$ & NS \\
DBP & $\mathrm{mm} \mathrm{Hg}$ & $83(14)$ & $79(13)$ & 0.0133 \\
heart rate & b.p.m. & $68(11)$ & $76(12)$ & $<0.0001$ \\
mean arterial pressure & $\mathrm{mm} \mathrm{Hg}$ & $98(16)$ & $94(16)$ & 0.0394 \\
pulse wave velocity & $\mathrm{m} \cdot \mathrm{sec}^{-1}$ & $6.86(1.74)$ & $7.33(1.90)$ & 0.0369 \\
\hline
\end{tabular}

Results expressed as means (1SD) or proportions (\%). DBP: diastolic blood pressure; HIV: human immunodeficiency virus; SBP: systolic blood pressure. NS: non-significant.

Table 5. Untreated vs treated HIV patients.

\begin{tabular}{|c|c|c|c|c|}
\hline & & untreated & treated & $p$ \\
\hline$n$ & & 108 & 130 & $\sim$ \\
\hline duration since HIV diagnosis & months & $19(28)$ & $46(30)$ & $<0.0001$ \\
\hline BMI & $\mathrm{kg} \cdot \mathrm{m}^{-2}$ & $24.0(4.5)$ & $24.9(4.3)$ & NS \\
\hline waist circumference & $\mathrm{cm}$ & $81(10)$ & $85(10)$ & 0.0024 \\
\hline waist $\cdot$ height $^{-1}$ & $\sim$ & $0.50(0.06)$ & $0.52(0.07)$ & 0.0201 \\
\hline hypertension & $\%$ & 41 & 55 & 0.0375 \\
\hline mean arterial pressure & $\mathrm{mm} \mathrm{Hg}$ & $91(15)$ & 97 (17) & 0.0046 \\
\hline cholesterol & $\mathrm{mg} \cdot \mathrm{dl}^{-1}$ & $151(47)$ & $176(48)$ & $<0.0001$ \\
\hline LDL-C & $\mathrm{mg} \cdot \mathrm{dl}^{-1}$ & 96 (39) & $108(36)$ & 0.0144 \\
\hline non-HDL-C & $\mathrm{mg} \cdot \mathrm{dl}^{-1}$ & 117 (39) & 126 (39) & NS \\
\hline HDL-C & $\mathrm{mg} \cdot \mathrm{dl}^{-1}$ & $34(17)$ & $51(20)$ & $<0.0001$ \\
\hline total-C·HDL-C ${ }^{-1}$ & $\sim$ & $6.71(6.98)$ & 3.94 (2.39) & 0.0001 \\
\hline non-HDL-C.HDL-C ${ }^{-1}$ & $\sim$ & $5.71(6.98)$ & $2.95(2.38)$ & 0.0001 \\
\hline $\log (\mathrm{TG}) \cdot \mathrm{HDL}-\mathrm{C}^{-1}$ & $\sim$ & $0.114(0.169)$ & $0.047(0.037)$ & $<0.0001$ \\
\hline
\end{tabular}

Results expressed as means (1SD) or proportions (\%). BMI: body mass index; C: cholesterol; HDL: high-density lipoprotein; HIV: human immunodeficiency virus; LDL: low-density lipoprotein; TG: triglycerides. NS: non-significant.

study population that was more pronounced, albeit non-significantly, in HIV-infected patients. Our data are higher than the $16.2 \%$ estimate in the general population in Sub-Saharan Africa, and also higher than the expected prevalence for a 40-year old Cameroonian population [21]. Our findings of a higher-than-expected rate in Cameroonian HIV-positive individuals are in keeping with previous observations from developed countries suggesting increased hypertension prevalence in HIV-positive individuals [11] [12] [22]. In this Cameroonian cohort, the burden of hypertension was more pronounced among treated HIV individuals, consistent with recent observations among HIV-treated patients from Kenya [23]. Higher salt intake is responsible for a substantial 
component of the rise in blood pressure with age [24], and is a major determinant of hypertension in Black individuals [25]. In the present study, the finding of a lower mean arterial pressure observed in Cameroonian HIVpositive individuals may partly be ascribed to lower salt intake and/or increased extrarenal sodium losses, as their urinary $\mathrm{Na}^{+}$concentration was significantly lower than that of Controls.

Impaired glucose tolerance and diabetes are major determinants of CVD risk [26], and are more prevalent in HIV-positive patients [11] [12] [27] [28], as confirmed by the present study. Our results are in keeping with recent observations in Caucasian HIV-positive individuals [11] [12] [27] [28], in whom acquired abnormal glucose homeostasis was mostly ascribed to ART, especially when PIs were present. In the present study, the underlying mechanism(s) form impaired glucose homeostasis cannot be further precised, although it should be emphasized that in subgroups analysis, prevalence of diabetes and of impaired fasting glucose were similar in treated or untreated HIV individuals.

Another relevant finding of the present study is that HIV infection was associated with a substantial degree of dyslipidemia, characterized by increased atherogenic ratios and atherogenic dyslipidemia, the hallmark of the latter being concurrent decreased HDL-C plus raised triglycerides. The role of both HDL-C [29] and triglycerides [30] in the pathophysiology of atherosclerosis has been clearly demonstrated, and recent findings show that atherogenic dyslipidemia increases CV risk even when LDL-C is not elevated and/or controlled with statins [31]. This suggests increased lipid-related, modifiable atherosclerosis risk in Cameroonian patients with HIV.

The MetS is a well-established modifiable determinant of new-onset diabetes, incident CV events and residual CV risk [12] [17] [32], and is more prevalent in the presence of HIV infection [10]-[12], as confirmed in the present study. Obesity is a major driver of the MetS in the general population [32] as well as in HIV-infected individuals [10]-[12] [23]. Inexpectedly, obesity prevalence and central adiposity indices, BMI and waist circumference in this study were all lower in HIV individuals vs Controls, in contrast with observations from Kenya reporting increased obesity in Black African HIV patients [23]. Such discrepancies may partly result from regional, environmental, as well as ethno-geographical differences between Cameroonian and Kenyan populations, the former being overwhelmingly from Bantu origin.

Despite lower indices for central adiposity in these Cameroonian patients, HIV infection was associated with a dramatic increase in MetS prevalence and in the severity of its score, the latter being a surrogate of wholebody insulin resistance. This is in agreement with recent data from HIV patients in developed countries [23] [32]. Wilst these MetS-related disturbances were more pronounced in untreated HIV individuals, the increased prevalence of MetS and of its individual components were previously reported mainly from Caucasian patients on ART containing PIs [11] [12]. Our results suggest a possible influence of environmental, socio-cultural and/or genetic factors, next to HIV infection itself and/or of its treatment, on MetS expression.

In the present study, HIV infection also represents a risk factor for early vascular ageing, as elicited by a higher aortic PWV, a direct measure of aortic stiffness. Arterial stiffening is an inevitable part of the ageing process [33] [34], since with advancing age the aorta and large arteries become progressively less distensible, with reduced ability to absorb pulsations from the ejecting ventricle [33]. The higher aortic stiffness observed in Cameroonian HIV individuals was consistent with recent reports in Caucasian patients [4] [5] [9] [14] [35]. Whilst the faster PWV in Caucasian patients was ascribed to both PIs use [8] [14] [35] and to HIV infection itself [9], in this study of Black native patients from Cameroon, PWV acceleration seems to be more linked to chronic infection or inflammation related to HIV status, together with the subsequent exposure to acquired conventional CV risk factors, such as hypertension, abnormal glucose homeostasis (IFG or diabetes) and dyslipidemia-hypercholesterolemia, all of which otherwise being well-established determinants of arterial stiffening [33] [34] [36]. Of special interest is the high prevalence of atherogenic dyslipidemia observed in the present study, which may contribute to increase arterial stiffness in HIV patients. Moreover, growing evidence indicates that chronic inflammation is associated with large artery stiffness, by promoting structural changes and/or endothelial dysfunction [7] [8] [37] [38]. Thus, chronic inflammation resulting from HIV infection may promote aortic stiffness in Cameroon HIV individuals, especially among the never-treated. Indeed, among HIV-infected participants, whereas MAP which is an established determinant of PWV [13] [20] was higher in treated (vs untreated) HIV individuals, their aortic PWV was slightly slower than that of never-treated patients (data not shown).

On the other hand, the present study shows that Cameroonian HIV-treated patients may derive some unexpected benefit from the vasculoprotective effect [29] of a relatively higher HDL-C level, as well as from the underutilization of PIs, financial constraints limiting access to those drugs for many Cameroonian patients. Indeed, the majority of those Cameroonian HIV-treated patients were on NNRT (data not shown) which has no impact 
on PWV, contrasting with Caucasian patients, whose ART often includes PIs [5] [8] [14] which increase arterial stiffness.

\section{Limitations}

The present study has several potential limitations, including a relatively reduced sample size, a transversal design, the use of surrogates markers for central fat assessment and insulin sensitivity, and the lack of measurement of subclinical inflammatory markers, such as highly-sensitive C-reactive protein (hsCRP) to name but a few. Further studies involving a larger-scale sample population and including assessment of relevant parameters (such as hsCRP, plasma RNA-HIH) are needed to confirm our findings. Due to financial and logistical constraints, we recruited only at one centre in Cameroon, and our results might therefore not be representative of other Black HIV patients in Cameroon or in sub-Saharan Africa. However, the strong differences in cardiometabolic phenotype and PWV between patients and Controls suggest that the conclusions of the present study are unlikely to result from study limitations.

\section{Conclusion}

HIV infection is associated with a high prevalence of mostly undiagnosed hypertension and abnormal glucose homeostasis, together with increased frequency and severity of the metabolic syndrome in Black African patients, alongside accelerated vascular wall damage, witnessed by higher aortic pulse wave velocity.

\section{Conflict of Interests}

None of the authors declared any conflict of interest as regards the content of this manuscript.

\section{Funding}

This work was supported by the French Foundation for research on arterial hypertension.

\section{References}

[1] Sabin, C.A., Worm, S.W., Weber, R., et al. (2008) Use of Nucleoside Reverse Transcriptase Inhibitors and Risk of Myocardial Infarction in HIV-Infected Patients Enrolled in the D: A: D Study: A Multi-Cohort Collaboration. Lancet, 371, 1417-1426. http://dx.doi.org/10.1016/S0140-6736(08)60423-7

[2] Corral, I., Quereda, C., Moreno, A., et al. (2009) Cerebrovascular Ischemic Events in HIV-1-Infected Patients Receiving Highly Active Antiretroviral Therapy: Incidence and Risk Factors. Cerebrovascular Diseases, 27, 559-563. http://dx.doi.org/10.1159/000214219

[3] Constans, J. and Conri, C. (2006) Circulating Markers of Endothelial Function in Cardiovascular Disease. Clinica Chimica Acta, 368, 33-47. http://dx.doi.org/10.1016/j.cca.2005.12.030

[4] Hsue, P.Y., Lo, J.C., Franklin, A., et al. (2004) Progression of Atherosclerosis as Assessed by Carotid Intima-Media Thickness in Patients with HIV Infection. Circulation, 109, 1603-1608. http://dx.doi.org/10.1161/01.CIR.0000124480.32233.8A

[5] Lorenz, M.W., Stephan, C., Harmjanz, A., et al. (2008) Both Long-Term HIV Infection and Highly Active Antiretroviral Therapy Are Independent Risk Factors for Early Carotid Atherosclerosis. Atherosclerosis, 196, 720-726. http://dx.doi.org/10.1016/j.atherosclerosis.2006.12.022

[6] Glazier, J.J., Spears, J.R. and Murphy, M.C. (2006) Interventional Approach to Recurrent Myocardial Infarction in HIV-1 Infection. Journal of Interventional Cardiology, 19, 93-98. http://dx.doi.org/10.1111/j.1540-8183.2006.00111.X

[7] Fourie, C., van Rooyen, J., Pieters, M., Conradie, K., Hoekstra, T. and Schutte, A. (2011) Is HIV-1 Infection Associated with Endothelial Dysfunction in a Population of African Ancestry in South Africa? Cardiovascular Journal of Africa, 22, 134-140. http://dx.doi.org/10.5830/CVJA-2010-056

[8] Fisher, S.D., Miller, T.L. and Lipshultz, S.E. (2006) Impact of HIV and Highly Active Antiretroviral Therapy on Leukocyte Adhesion Molecules, Arterial Inflammation, Dyslipidemia, and Atherosclerosis. Atherosclerosis, 185, 1-11. http://dx.doi.org/10.1016/j.atherosclerosis.2005.09.025

[9] Schillaci, G., De Socio, G.V., Pucci, G., et al. (2008) Aortic Stiffness in Untreated Adult Patients with Human Immunodeficiency Virus Infection. Hypertension, 52, 308-313. http://dx.doi.org/10.1161/HYPERTENSIONAHA.108.114660 
[10] Aberg, J.A. (2009) Cardiovascular Complications in HIV Management: Past, Present, and Future. Journal of Acquired Immune Deficiency Syndromes, 50, 54-64. http://dx.doi.org/10.1097/QAI.0b013e31818ceaa4

[11] Feitas, P., Carvalho, D., Souto, S., et al. (2011) Impact of Lipodystrophy on the Prevalence and Components of Metabolic Syndrome in HIV-Infected Patients. BMC Infectious Diseases, 11, 246. http://dx.doi.org/10.1186/1471-2334-11-246

[12] Lakka, H.M., Laaksonen, D.E., Lakka, T.A., et al. (2002) The Metabolic Syndrome and Total and Cardiovascular Disease Mortality in Middle-Aged Men. Journal of the American Medical Association, 288, 2709-2716. http://dx.doi.org/10.1001/jama.288.21.2709

[13] Hansen, T.W., Staessen, J.A., Torp-Pedersen, C., et al. (2006) Prognostic Value of Aortic Pulse Wave Velocity as Index of Arterial Stiffness in the General Population. Circulation, 113, 664-670. http://dx.doi.org/10.1161/CIRCULATIONAHA.105.579342

[14] Lekakis, J., Ikonomidis, I., Palios, J., et al. (2009) Association of Highly Active Antiretroviral Therapy with Increased Arterial Stiffness in Patients Infected with Human Immunodeficiency Virus. American Journal of Hypertension, 22, 828-834. http://dx.doi.org/10.1038/ajh.2009.90

[15] Joint United Nations Programme on HIV/AIDS (UNAIDS) (2008) Report on the Global HIV/AIDS Epidemic. Geneva. http://www.unaids.org/en/KnowledgeCentre/HIVData/GlobalReport/2008/2008_Global_report.asp

[16] Mancia, G., De Backer, G., Dominiczak, A., et al. (2007) 2007 Guidelines for the Management of Arterial Hypertension: The Task Force for the Management of Arterial Hypertension of the European Society of Hypertension (ESH) and of the European Society of Cardiology (ESC). Journal of Hypertension, 25, 1105-1187. http://dx.doi.org/10.1097/HJH.0b013e3281fc975a

[17] Grundy, S.M., Cleeman, J.I., Daniels, S.R., et al. (2005) Diagnosis and Management of the Metabolic Syndrome: An American Heart Association/National Heart, Lung, and Blood Institute Scientific Statement. Circulation, 112, 27352752. http://dx.doi.org/10.1161/CIRCULATIONAHA.105.169404

[18] Hermans, M.P., Ahn, S.A. and Rousseau, M.F. (2010) $\log$ (TG)/HDL-C Is Related to Both Residual Cardiometabolic Risk and $\beta$-Cell Function Loss In type 2 Diabetes Males. Cardiovascular Diabetology, 9, 88. http://dx.doi.org/10.1186/1475-2840-9-88

[19] Asmar, R., Benetos, A., Topouchian, J., et al. (1995) Assessment of Arterial Distensibility by Automatic Pulse Wave Velocity Measurement. Validation and Clinical Application Studies. Hypertension, 26, 485-490. http://dx.doi.org/10.1161/01.HYP.26.3.485

[20] Lemogoum, D., Van Bortel, L., Najem, B., et al. (2004) Arterial Stiffness and Wave Reflections in Patients with Sickle Cell Disease. Hypertension, 44, 924-929. http://dx.doi.org/10.1161/01.HYP.0000148506.73622.ba

[21] Twagirumukiza, M., De Bacquer, D., Kips, J., de Backer, G., Vander Stichele, R. and Van Bortel, L. (2011) Current and Projected Prevalence of Arterial Hypertension in Sub-Saharan Africa by Sex, Age and Habitat: An Estimate from Population Studies. Journal of Hypertension, 29, 1243-1252. http://dx.doi.org/10.1097/HJH.0b013e328346995d

[22] Palacios, R., Santos, J., García, A., Castells, E., González, M., Ruiz, J. and Márquez, M. (2006) Impact of Highly Active Antiretroviral Therapy on Blood Pressure in HIV-Infected Patients. A Prospective Study in a Cohort of Naive Patients. HIV Medicine, 7, 10-15. http://dx.doi.org/10.1111/j.1468-1293.2005.00333.x

[23] Bloomfield, G.S., Hogan, J.W., Keter, A., Sang, E., Carter, E.J., Velazquez, E.J. and Kimaiyo, S. (2011) Hypertension and Obesity as Cardiovascular Risk Factors among HIV Seropositive Patients in Western Kenya. PloS ONE, 6, Article ID: e22288. http://dx.doi.org/10.1371/journal.pone.0022288

[24] He, F.J. and MacGregor, G.A. (2009) A Comprehensive Review on Salt and Health and Current Experience of Worldwide Salt Reduction Programmes. Journal of Human Hypertension, 23, 363-384. http://dx.doi.org/10.1038/jhh.2008.144

[25] He, F.J., Marciniak, M., Visagie, E., Markandu, N.D., Anand, V., Dalton, R.N. and MacGregor, G.A. (2009) Effect of Modest Salt Reduction on Blood Pressure, Urinary Albumin, and Pulse Wave Velocity in White, Black, and Asian Mild Hypertensives. Hypertension, 54, 482-488. http://dx.doi.org/10.1161/HYPERTENSIONAHA.109.133223

[26] Yoshida, M., Mita, T., Yamamoto, R., Shimizu, T., Ikeda, F., Ohmura, C., Kanazawa, A., Hirose, T., Kawamori, R. and Watada, H. (2012) The Combination of Framingham Risk Score and Carotid Intima-Media Thickness Improves the Prediction of Cardiovascular Events in Patients with Type 2 Diabetes. Diabetes Care, 35, 178-180. http://dx.doi.org/10.2337/dc11-1333

[27] Brown, T.T., Cole, S.R., Li, X., Kingsley, L.A., Palella, F.J., Riddler, S.A., Visscher, B.R., Margolick, J.B. and Dobs, A.S. (2005) Antiretroviral Therapy and the Prevalence and Incidence of Diabetes Mellitus in the Multicenter AIDS Cohort Study. JAMA Internal Medicine, 165, 1179-1184. http://dx.doi.org/10.1001/archinte.165.10.1179

[28] Capeau, J., Bouteloup, V., Katlama, C., et al. (2012) Ten-Year Diabetes Incidence in 1046 HIV-Infected Patients Started on a Combination Antiretroviral Treatment: The ANRS CO8 APROCO-COPILOTE Cohort. AIDS, 26, 303-314. http://dx.doi.org/10.1097/QAD.0b013e32834e8776 
[29] Mineo, C., Deguchi, H., Griffin, J.H. and Shaul, P.W. (2006) Endothelial and Antithrombotic Actions of HDL. Circulation Research, 98, 1352-1364. http://dx.doi.org/10.1161/01.RES.0000225982.01988.93

[30] Patel, S., Puranik, R., Nakhla, S., Lundman, P., Stocker, R., Wang, X.S., Lambert, G., Rye, K.A., Barter, P.J., Nicholls, S.J. and Celermajer, D.S. (2009) Acute Hypertriglyceridaemia in Humans Increases the Triglyceride Content and Decreases the Anti-Inflammatory Capacity of High Density Lipoproteins. Atherosclerosis, 204, 424-428. http://dx.doi.org/10.1016/j.atherosclerosis.2008.07.047

[31] Cziraky, M.J., Watson, K.E. and Talbert, R.L. (2008) Targeting Low HDL-Cholesterol to Decrease Residual Cardiovascular Risk in the Managed Care Setting. Journal of Managed Care Pharmacy, 14, S3-S28.

[32] Isomaa, B., Almgren, P., Tuomi, T., Forsén, B., Lahti, K., Nissén, M., Taskinen, M.R. and Groop, L. (2001) Cardiovascular Morbidity and Mortality Associated with the Metabolic Syndrome. Diabetes Care, 24, 683-689. http://dx.doi.org/10.2337/diacare.24.4.683

[33] Greenwald, S., Carter, A. and Berry, C. (1990) Effect of Age on the in Vitro Reflection Coefficient of the Aortoiliac Bifurcation in Humans. Circulation, 82, 114-123. http://dx.doi.org/10.1161/01.CIR.82.1.114

[34] Avolio, A.P., Deng, F.Q., Li, W.Q., Luo, Y.F., Huang, Z.D., Xing, L.F. and O’Rourke, M.F. (1985) Effects of Aging on Arterial Distensibility in Populations with High and Low Prevalence of Hypertension: Comparison between Urban and Rural Communities in China. Circulation, 71, 202-210. http://dx.doi.org/10.1161/01.CIR.71.2.202

[35] van Vonderen, M.G., Smulders, Y.M., Stehouwer, C.D., et al. (2009) Carotid Intima-Media Thickness and Arterial Stiffness in HIV Infected Patients: The Role of HIV, Antiretroviral Therapy, and Lipodystrophy. Journal of Acquired Immune Deficiency Syndromes, 50, 153-161. http://dx.doi.org/10.1097/QAI.0b013e31819367cd

[36] Zieman, S.J., Melenovsky, V. and Kass, D.A. (2005) Mechanisms, Pathophysiology, and Therapy of Arterial Stiffness. Arteriosclerosis, Thrombosis, and Vascular Biology, 25, 932-943. http://dx.doi.org/10.1161/01.ATV.0000160548.78317.29

[37] McEniery, C.M. and Wilkinson, I.B. (2005) Large Artery Stiffness and Inflammation. Journal of Human Hypertension, 19, 507-509. http://dx.doi.org/10.1038/sj.jhh.1001814

[38] Hingorani, A.D., Cross, J., Kharbanda, R.K., et al. (2000) Acute Systemic Inflammation Impairs Endothelium-Dependent Dilatation in Humans. Circulation, 102, 994-999. http://dx.doi.org/10.1161/01.CIR.102.9.994 